\title{
Evolution of Interaction in Physically-based Modelling
}

\author{
M. Bordegoni, U. Cugini and F. De Angelis \\ Dipartimento di Ingegneria Industriale \\ Università di Parma - Parco Area delle Scienze, 181/A \\ I-43100 PARMA-Italy \\ e-mail: [mb, cugini, fda]@ied.unipr.it
}

\begin{abstract}
This paper outlines new trends in geometric modelling, showing how systems are moving from a geometry-based approach to a physically-based approach. The possibility to simulate the actual behaviour of a product is at the basis of Virtual Prototypes that are becoming a common practice in today's product development process. As a consequence, also interaction modalities and techniques have to be improved in order to satisfy new system requirements and functionalities. The research work described in this paper shows how haptic interaction techniques represent an evolution of current interaction technologies providing intuitive and realistic modalities for interacting with virtual applications. In particular, the paper describes a research work we have carried out integrating haptic technologies together with physically-based modelling and simulation techniques.
\end{abstract}

Keywords: haptic interaction, physically-based modelling, non-rigid material modelling, virtual prototyping, human-computer interaction.

\section{INTRODUCTION}

This paper addresses issues related to new evolution trends in geometric modelling and CAD systems, focusing on physically-based modelling and haptic interaction.

In current geometric modelling systems, supporting both solid and surface modelling, the shape of the objects is constant and nondeformable. Basically, objects are described by means of their geometry and topology, and modifications can be performed by roto-traslation transformations applied through their graphical representation. Physical properties of the objects are mostly considered as attributes associated with the geometry within the model data structure. 
As we can notice everyday, the world is not made of just rigid objects. Actually, the objects shape can change, and that may depend on several factors like the previous objects status, on forces exerted on the objects, on constraints, etc. Therefore, the objects shape can be described as a function of time and of internal and external context:

\section{Shape $=f($ time, int/ext context $)$}

In order to take into account all these aspects, the trend of modelling is about to move from a geometry-based approach to a physically-based approach, where geometry is not the only piece of information defining an object (Cugini, 1999). In physically-based modelling, the object description includes a set of physical characteristics, such as mass, speed, viscosity, etc. Besides, it is possible to model external forces acting on the object, obstacles the object interacts with, collisions between object parts, etc.

Several research works have been presented and published recently on topics related to physically-based modelling (Cugini, 1999), (SIGGRAPH, 1999), underlying new technological trends and expectations the users have about these new modelling features. In fact, physically-based modelling applies to several fields, going from industrial sectors (like automotive, food, clothing industry) to medical field (Cugini, 1999) (Rizzi, 2000) (Westwood, 1999).

The possibility of simulating the actual behaviour of the products, beyond their geometry, is at the basis of Digital Mock-Ups (DMU) and Virtual Prototypes. These are complex virtual models of products that enclose information so that virtual prototypes can be used in place of/together with physical mock-ups. Visual technology is today sufficiently advanced, and the rendering of DMUs can be very realistic. In fact, visual rendering techniques have evolved and improved in the last decades, offering very good and realistic representations of models. Conversely, human computer interaction techniques have not progressed much in the last two decades. Because of fast evolving and spreading of use of 3D model representations, we aspect that consequently interaction modalities and media evolve in more suitable techniques, that satisfy the requirements of these new applications.

Let us briefly analyse how human-computer interaction is performed in today's system and which is the trend for future communication modalities. 
Currently, human-computer interaction is mainly based on the WIMP (Window-Icon-Mouse-Pointer) paradigm where communication between user and computer occurs through devices like the display screen and the mouse. If we consider geometric modelling applications, we notice that there are several spaces where models and their representation exist (Figure 1). Geometric models have a 3D geometric description given in a 3D Geometric Modelling Space. 3D geometric models are mapped into a 2D representation -in a $2 D$ Representation Space- in order to be displayed to the user on a flat monitor.

Most of the systems support the representation of the functional relations among geometric objects. It is possible to define a family of possible shapes that satisfy some relational rules. These are used for defining an object instance obtained assigning some values to a set of functional parameters -in a Functional Representation Space. The user is interested to see both the entity and the parameters defining that geometry, and the relations among the geometries. This is done, for example, showing a feature tree in the 2D Representation Space, that is a symbolic structure representing the relations among the objects.

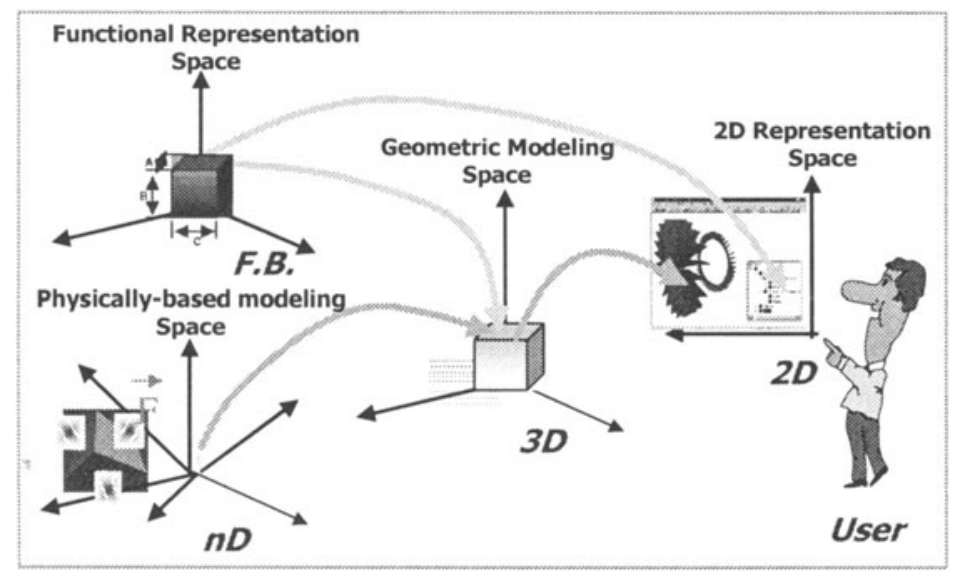

Figure 1. Model and Representation Spaces. Interaction flow from the system to the user.

More advanced systems support physically-based modelling techniques (Cugini, 1999). Object shape is anyway represented as a sequence of images on the flat monitor.

The fact that the user interacts with 3D models through their 2D representation might cause problems in correctly perceiving and disambiguating the objects represented (Faconti, 2000). 
Conversely, let now see how the user interact with models (Figure 2). Today, the user mainly interacts with models through their representation shown on the flat screen, pointing at the object, or at part of it to be manipulated, using a mouse. We can consider two types of interaction. One type of interaction is meant to be performed on the object representation with the only purpose to change its representation (zoom in/out, pan, change of viewpoint, etc.). A second type of interaction concerns the modification of the model, that has to be done through the modification of its representation on the screen.

In the case of functional models, the interaction on geometric elements is integrated with the interaction through the schematic representation of the relation tree, that is a 2D symbolic representation that allows the user to modify the relations among the parts, effecting then the representation of the object shape. While the interaction with the physically-based model of an object is not directly supported in today's systems.

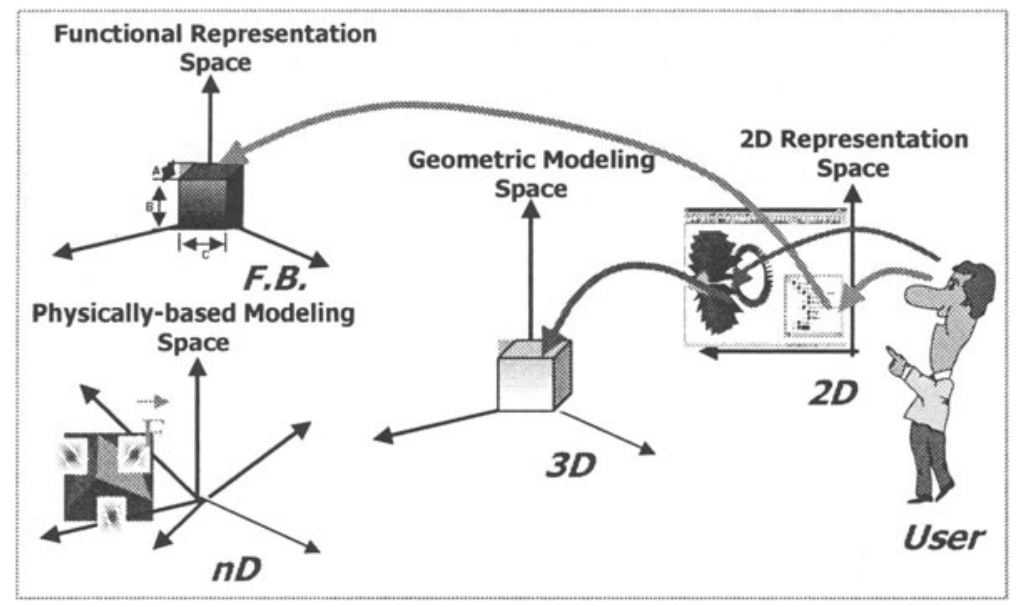

Figure 2. Interaction flow from the user to the system.

In order to make virtual prototypes completely efficient and effective, the users should be able to directly and intuitively interact with the model, as $\mathrm{s} /$ he is used to do with physical mock-ups. This implies that it should be possible to touch the product, disassemble its parts, check its behaviour, weight, deformability, etc. And the best would be to be able to do all that without considering the intermediate steps related to the geometric and functional models seen so far.

This type of interaction modality is not completely available today. In fact, most advanced current systems, so called Virtual Reality systems, 
mainly support the navigation within the virtual models, through interaction provided by sophisticated display devices (head-mounted display, goggles, etc.), and 3D input devices like spaceballs and gloves. Some new devices, named haptic devices, have been recently introduced in order to add realism to the interaction, joining haptic perception to the common visual one (SIGGRAPH, 1998). The implementation of haptic interaction with rigid objects is supported by device libraries (SansAble). The current research interest is in integrating haptic interaction with physically-based modelling. These devices would give a complementary support to the overall perception of the object (Figure 3). This new interaction technology and modality support users in perceiving physical properties of the objects modelled (it is soft, the surface is rough, etc.), and it would help users in solving ambiguous situations given by the 2D representation of 3D models (Faconti, 2000). Besides, it is a first step allowing users to use their natural skill they have when interacting with the physical worlds, also when they interact with the digital world (Bordegoni, 1998).

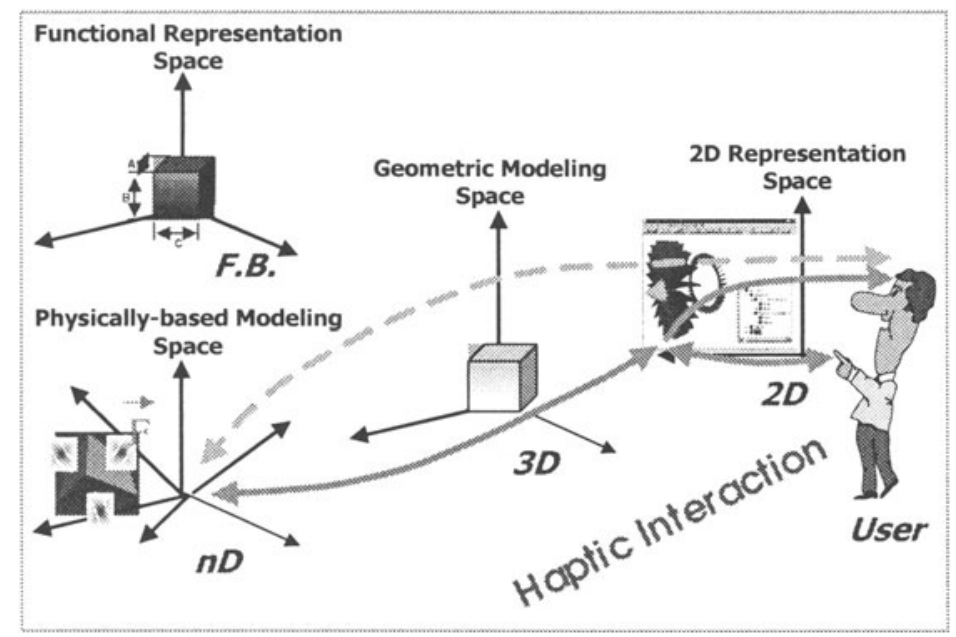

Figure 3. New trend in human-computer interaction: haptic interaction.

In this paper we show how the introduction of these new trends physically-based modelling and haptic interaction- are the leading edge of the development of future generation of CAD and geometric modelling systems. The following sections of this paper present the approach we have followed for simulating in real-time the behaviour of non-rigid objects, so as to be able to support haptic interaction. 


\section{HAPTIC RENDERING OF NON-RIGID OBJECTS}

The main topic of our research concerns the integration of haptic interaction and physically-based modelling.

We have developed a non-rigid material simulator (NRM Simulator) for modelling and simulating non-rigid materials behaviour (Denti, 1996). The environment allows us to describe non-rigid objects through a discrete model, known as particle-based model. An object is described by a set of particles distributed in space and related by means of internal forces and constraints. Depending on the type of material to be simulated, different discretization rules and force distributions are used. The environment also allows us to define and simulate some external forces, constraints and obstacles interfering with the objects. In this way, we are able to simulate the behaviour of a non-rigid object when grasped and manipulated.

In order to improve realism of interaction with physically-based models, we have explored the possibility of using haptic devices. Before going into details in the description of our work, we shortly introduce what haptic interaction is. Haptic interaction is an interaction modality based on the use of haptic devices that augments human-computer interaction with the sense of touch. Haptic devices are input/output devices allowing users wearing them to feel a realistic tactile representation of $3 \mathrm{D}$ objects.

Haptic interaction is based on haptic rendering, that is the tactile counterpart of graphic rendering. The basic idea of haptic rendering is quite simple. A positional transducer measures the current position of a user's finger, the position is compared against the objects defined in the virtual scene, and if an interference is detected the proper reaction force is computed and sent to an actuator acting on the user's finger. This must be repeated in a loop running at a convenient frequency. Previous work on haptic rendering has showed that the tactile perception is much more sensitive than the sight with respect to the minimum time interval needed to distinguish two different perceptions, hence while an image refresh rate of $30 \mathrm{~Hz}$ as used in the movies is enough to cheat the eye, a force refresh rate of about $1000 \mathrm{~Hz}$ appeared to be necessary to perform realistically haptic rendering (Massie, 1993). Figure 4 shows the haptic rendering loop of one of the most common haptic devices, the PHANToM device (SensAble), we have used in our experiments. 


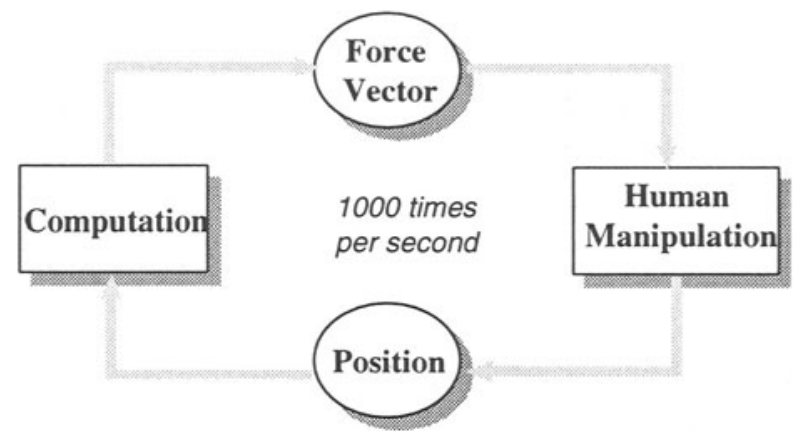

Figure 4. PHANToM haptic rendering loop.

Running the haptic rendering loop shown uninterruptedly at a rate of 1000 times per second is the main source of difficulties in haptic rendering. One intrinsic problem is that the typical time slice duration of an operating system is in the range $50-200 \mathrm{~ms}$, which means that whenever the process or thread running the rendering loop loses a time slice due to multitasking, it also loses about a hundred loop cycles, which makes the resulting haptic rendering unacceptable. Multitasking, however, cannot be avoided as we normally want the haptic rendering to be augmented by interactively displaying the manipulated scene, and also the operating system always has its own processes running in background. Satisfying solutions to this problem include using multiprocessor computers and/or modifying the operating system scheduling policy, or using dedicated hardware running the rendering loop on its own.

Besides that, the major problem encountered is that running the loop at $1000 \mathrm{~Hz}$ means that the time available for the computation is less than $1 \mathrm{~ms}$, which is enough for rigid object simulation, but is not enough for non-rigid object simulation, at least using our non-rigid material Simulator on currently available PC hardware.

Hereafter, we describe some results of experiments we have made to estimate the ratio between available and required computational power to perform this kind of simulation (De Angelis, 1997).

\subsection{Integration of haptic rendering with a pre-existing NRM Simulator}

The computational cost of simulating a non-rigid object using our NRM Simulator depends on the number of particles describing the object, but also on other variables, like the constraints among the particles, the 
type and number of internal forces used and the stiffness of the material, just to mention some. While this implies that it is not appropriate in general to describe the computational cost of the Simulator as an $O(n)$ function where $n$ is the number of particles, it is however possible to get a qualitative idea of that function by performing some measurements on a test case where all variables except $n$ are kept as much as possible constant.

A test has been executed on different objects having a number of particles equal to $i^{3}$ for $i=2,3,4, \ldots$, i.e. regularly discretized cubes having $2,3,4, \ldots$ particles along their side.

For each object, the average time for one iteration was determined; the results are reported in Figure 5. The tests were executed on an DEC Alphastation 500 running at $400 \mathrm{MHz}$.

As it can be seen, most of the diagram is dramatically above the $1 \mathrm{~ms}$ value, and it is also non linear. However, the test setup was based on a quite accurate physical model while less accurate simulations run faster. Additional tests run with a simplified setup led to better results; the best result for a very simplified model made of 4 particles was 0.4 ms per iteration. We can conclude that a computer faster by 2 or 3 times would probably have accomplished the task, but the simulated object was made by just 4 particles while real applications require hundreds or thousands of particles to properly represent the given objects. Even taking into account that computer speed keeps doubling every 18 months (Moore's law), this approach won't be useful for real applications for several years.

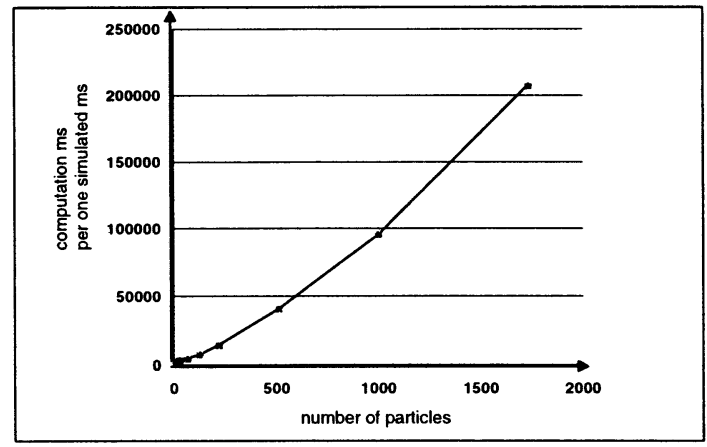

Figure 5. Computational time as a function of the number of particles on an Alphastation 500 running at $400 \mathrm{MHz}$. 


\subsection{Off-line simulation}

As discussed above, the simulation run using the pre-existing physically-based simulator (Denti, 1996) is limited by the computational cost of the simulation. Running the rendering loop at $1000 \mathrm{~Hz}$ requires to perform the computation in just one thousandth of a second, and the most complex object we were able to simulate in such a short time using our currently available hardware was a highly simplified model made by just four particles, where "highly simplified" means that most of the simulator capabilities were disabled.

A workaround to this limitation, which we name off-line simulation, consists of performing the simulation before the haptic rendering and to just use computation results at run-time. This was successfully implemented on special cases, like some object geometries having particular symmetry properties exhibiting the same physical behavior in every point of their surface, if they are made of a homogeneous and isotropic material. Examples of such geometries are spheres, the halfspace. If a random point on the surface of a soft sphere is pressed, the behavior (shape deformation and reaction forces) is exactly the same as any other point of its surface, being a function of the depth of the penetration relative to the surface shape in rest conditions. The same observation also applies to the halfspace and, with reasonable approximation, to other object geometries like "long" cylinders and "large", and "flat" boxes, if the interaction happens far enough from the ends or edges.

The basic idea of this experiment is that, as in all these cases the object reaction is a function independent of the actual point where the interaction takes place, depending only on the penetration depth of the interaction point, it is possible to pre-compute this function once, and apply the results wherever the actual interaction happens. Hence, the computation is divided into two different stages: off-line computation and run-time computation. This part of the computation can be done using a true physically-based simulator, with no constraints on computational time. A random point on the chosen object surface is pushed towards the inner of the object, along a direction normal to the surface and at a speed low enough to make the contribution due to dynamics and damping negligible. During the simulation, data are collected for later run-time rendering. A finite set of positions (only the penetration depth is significant) is fixed prior to the simulation, and whenever one of these positions is reached during the simulation, data relative to the reaction force and the surface deformation are registered. At run time, the actual 
position of the user is read, the corresponding penetration depth is determined and is compared with the set of known depths used in the offline simulation phase. The actual reaction force and surface deformation are determined by interpolation. Figure 6 shows two screenshots of an execution of the program. The little sphere represents the user's finger position in the virtual scene; the user is exerting different pressure in different points of the halfspace.
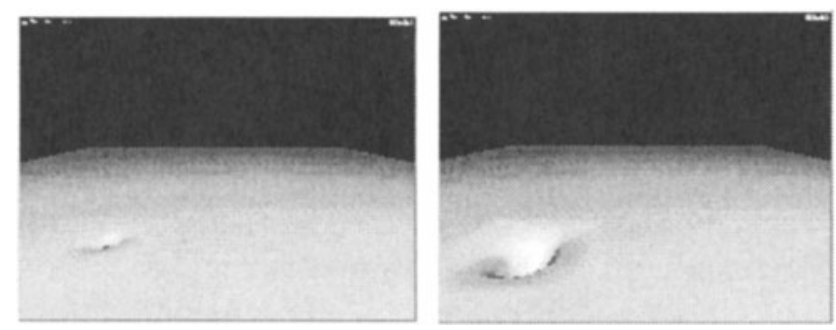

Figure 6. Soft halfspace deformation simulated using pre-computed data.

The results were encouraging: the combination of tactile and visual stimuli was quite "realistic" and anybody who tried the application shared the same opinion.

However, there are some observations on the generality of the approach. Other objects having special geometrical properties, like the sphere, could have been easily implemented. More objects, having less special properties, could also have been handled under some conditions, like "long" cylinders or pipes being manipulated far enough from the ends, or "large" boxes being manipulated far enough from the edges. But there is no straightforward solution for arbitrary geometries.

The strategy of performing the simulation once, and applying the results anywhere the interaction happens, would not work with arbitrary geometries as their behavior changes from point to point. Performing a number of off-line simulations in different parts of the object may seem feasible, but the amount of pre-computed data would grow proportionally to the number of sampled points, and this would also lead to expensive $\mathrm{N}$ dimensional interpolation at run-time.

Multi-finger manipulation is another problem if this approach is used. When more than one finger is used to interact with the object, the resulting deformation and forces cannot always be expressed as a combination of the deformations and forces due to the single fingers. Examples of such manipulations are when the user squeezes the object with two objects, or pinches it. 
Moreover, this approach can only deal with local deformations of the objects, but cannot handle deformations that are global or depend on the history of the manipulation. Examples of such manipulations are bending a pipe, or making a knot.

Due to these observations it follows that this approach has a limited application domain hence suffers of lack of generality.

\section{REAL-TIME SIMULATION OF HAPTIC DATA}

The experiment described in this session is based on the conclusions of the two former ones, the first one concerning direct integration of a pre-existing NRM Simulator with the haptic technology and the second one concerning off-line pre-computation of the haptic data.

We have seen that if the $1000 \mathrm{~Hz}$ rendering paradigm is followed strictly and a complete physically-based model is used, then real-time simulation is not possible yet. We have also seen that the off-line precomputation approach lacks of generality and can be successfully applied only to a few special cases. Furthermore, it can be observed that human manipulation is mostly a qualitative process; hence a NRM Simulation for haptic rendering is not required to supply exact data.

According to these observations, the basic idea of this last experiment is to implement an approximated, but fast, NRM simulator, and to perform the haptic rendering even though the $1000 \mathrm{~Hz}$ frequency is not reached. According to this approach the simulation is always tied to the real-time, and the precision depends on the computational power. The faster the computer is, the more accurate the results are. In this way we can today perform the real-time simulation on our currently available hardware, though the accuracy is poor.

Real-time simulation of haptic data is achieved by:

a) Overcoming the 1 thousandth of a second time limit for the computation. The haptic rendering loop is now asynchronous to the simulation loop, and follows the computation with a certain delay by interpolating the results that are supplied at a lower frequency (figure 7). The time available for the computation in this case is higher of a factor equal to the number of milliseconds of delay. For example, a delay of 30 milliseconds, almost imperceptible, increases of 30 times the available computing time. As demonstrated in some research work, the tactile perception relies on the images received by the eye. The same finger stimulation can be perceived as different shapes depending on the images displayed on the monitor. At a typical image refresh rate used in 
TV/cinema, which is $30 \mathrm{~Hz}$, the same image is displayed for $33 \mathrm{~ms}$ long, and we don't mean to go any faster to save CPU cycles for the simulation. According to that, it is "useless" to change the force vector output by the haptic device before $33 \mathrm{~ms}$, as the eye is still receiving the same image.

b) Developing new software for approximated simulation. Running a high-fidelity simulator with most of its features turned off is useless and much slower than simpler software that just lacks those features. The high-fidelity simulator was written before that real-time needs arose, with no computational time constraints, having accurate results as the main goal. The high-speed approximate simulator that is currently being developed has speed as the only purpose -in order to support real-time haptic interaction- and whenever a trade off is possible between performances and accuracy, the choice is for speed. This simulator is still particle-based and using a spring-damper approach.

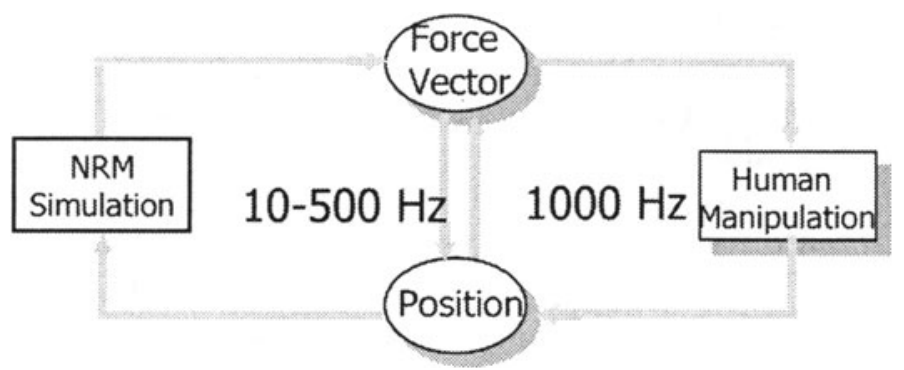

Figure 7. Asynchronous rendering model.

A prototype of a simulator implementing these concepts is running and is able to process a 1000 particles model from 20 to 50 times per second on normal PC hardware (20 Hz on a P-133, $50 \mathrm{~Hz}$ on a PPro-200). This allows the approximation of real-time haptic rendering of the model, by introducing a short delay as described above. As faster computers will be available, the model processing frequency for a given number of particles will increase, thus allowing shorter delays and more accurate results.

An example of haptic interaction using our prototype is shown in Figure 8. The figure shows a sequence of screenshots from the execution of the simulator. The user is interacting in real-time with a simulated soft cube using a PHANToM device. The sphere represents the user's finger. The experiment implementation reached the expected results. Real-time haptic rendering was possible at the expense of completeness of physical model, but generality has been preserved in that no assumptions have 
been made on the simulated models. The approach has showed to be feasible, and the obtained performances to be satisfying.

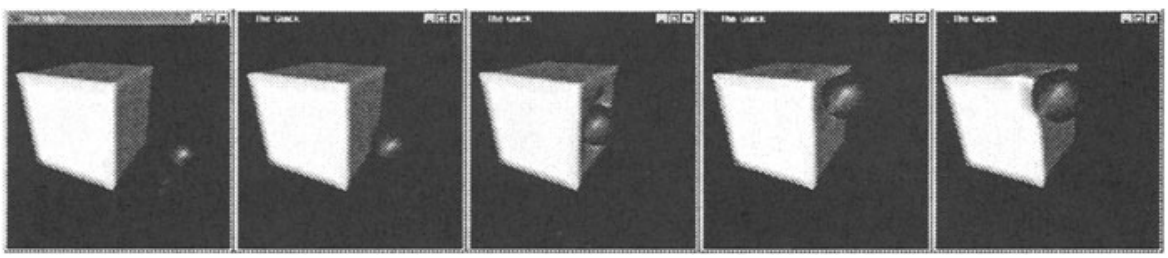

Figure 8. Example of haptic interaction with a soft cube.

\section{CONCLUSIONS}

This paper has presented some considerations about the evolution of human-computer interaction according to state-of-the-art systems and technology. It has been argued that systems are moving from a geometrybased to a physically-based approach. In that, geometry is not the only piece of information defining an object, but aspects concerning physical characteristics of the object have to be considered and included into the model. We have also argued that as a consequence of this change, the interaction between user and system requires to change accordingly. Devices providing force feedback have appeared recently, allowing the users to perceive physical features of the objects in an intuitive way.

In the second part of the paper we have described a system prototype we have developed that aims at coupling haptic interaction and physically-based modelling, into a real-time simulation environment. The prototype developed is running and performs multi-finger manipulation of non-rigid objects. It is currently being used for modelling and testing the realism of virtual models of non-rigid products.

\section{REFERENCES}

Bordegoni M. and Cugini U. (1998). Analysis of Skill for Robotics Manipulators through Human Demonstration and Haptic Interaction, Sixth IASTED International Conference ROBOTICS AND MANUFACTURING, Banff, Canada.

Cugini, U., Bordegoni, M., Rizzi, C., De Angelis, F., and Prati, M. (1999). Modelling and Haptic Interaction with non-rigid materials. Eurographics'99 - State-of-the-Art Reports, Milano, Italy.

De Angelis F., Bordegoni M, Frugoli G., Rizzi C. (1997). Realistic Haptic Rendering of Non-rigid Objects, in Proceedings of The Second PHANToM User Group Workshop, MIT, Cambridge, MA. 
Denti P., Dragoni P., Frugoli G., Rizzi C. (1996). SoftWorld: A system to simulate nonrigid products behavior in industrial processes, in Proceeding ESS 1996, Vol. II, Genoa, Italy.

Faconti, G., Massink, M., Bordegoni, M., De Angelis, F. and Booth, S. (2000). Haptic Cues for Image Disambiguation. Computer Graphics Forum. Blackwell, 19(3), pp. 169-178, 2000.

Witkin A. et al. (1995). "Physically based modeling." In SIGGRAPH 95 Course Notes (Los Angeles, California, USA, Aug. 8-10), no. 34.

Massie T.H. (1993). Initial Haptic Explorations with the Phantom: Virtual Touch Through Point Interaction, B.S. Electrical Engineering Massachusetts Institute of Technology.

SIGGRAPH (1999). Physically Based Modeling. Course Note n. 36, SIGGRAPH'99.

SIGGRAPH (1998). Physical Interaction: The Nuts and Bolts of Using Touch Interfaces with Computer Graphics Applications. Course Notes n. 1, SIGGRAPH'98.

Rizzi C., Bordegoni M., Frugoli G. (2000). Simulation of non-rigid materials handling. Robot manipulation of deformable objects, by Dominik Henrich and Heinz Woern (Eds.), Advanced Manufacturing Series, Springer, pp. 199-210.

SensAble Technologies, Inc., URL: www.sensable.com.

Westwood J., ed. (1999). Medicine Meets Virtual Reality, no. 7 in MMVR, IOS Press. 